\title{
Causal Attribution Habits and Cultural Orientation as Contributing Factors to Students' Self-Efficacy: A Comparison Between Female Students in the United States and Saudi Arabia
}

\author{
Kerstin Hamann ${ }^{1}$, Ronan L.H. Wilson ${ }^{2}$, Bruce M. Wilson ${ }^{1}$ Maura A. E. Pilotti ${ }^{3}$ \\ ${ }^{1}$ School of Politics, Security, and International Affairs, University of Central Florida, USA, \\ ${ }^{2}$ Department of Psychology, University of South Florida, USA, ${ }^{3}$ College of Sciences and \\ Human Studies, Prince Mohammad Bin Fahd University, KSA.
}

\begin{abstract}
In the present research, we examined the extent to which explanations of desirable or undesirable outcomes (grades) can account for the self-efficacy of female college students from two societies with dissimilar cultural traditions: The United States of America, which is characterized by a dominant individualistic culture, and the Kingdom of Saudi Arabia, which has a dominant collectivist culture. A matched-pairs design ( $N=560 ; 280$ matched pairs) was used to ensure that students' self-efficacy levels were equated between cultural groups. We found cultural differences in the choice of explanations and in the extent to which explanations contribute to self-efficacy values. These findings are relevant to the development of curricula and instructional methods that are intended to prepare students from different cultures for academic success.
\end{abstract}

Keywords: Self-efficacy; causal attribution; cultural orientation. 


\section{Introduction}

One of the most important contributors to students' success is self-efficacy, a psychological trait that denotes a general confidence in one's abilities to overcome challenges and solve problems (Bandura, 1977; Majer 2009; Bartimote-Aufflick et al., 2016; Roick \& Ringeisen 2017). A review conducted by Bartimote-Aufflick et al. (2016) identified value, intrinsic motivation, learning strategy use, locus of control, and self-regulation as the factors most highly correlated with students' self-efficacy (see also van Dinther et al. 2011). While studies in the field of education have explored cultural differences between countries, the present research offers a novel contribution in that it collects individual-level data on culture instead of applying a country's values to the individual (see Bartimore-Aufflick et al., 2015) and includes an understudied population for comparison (female students from the Kingdom of Saudi Arabia, KSA).

Self-efficacy is a universal construct that applies across cultures although group differences exist. For instance, Scholz et al. (2002) found that individuals from collectivist cultures (e.g., Asia) have lower self-efficacy than those from individualistic cultures. There are also differences in how individuals explain actions and outcomes (i.e., causal attribution habits; Choi et al., 1999). It is unclear, however, to which extent causal attribution habits for desirable or undesirable outcomes may shape students' self-efficacy (Bandura, 1989) when self-efficacy does not differ between cultural groups. That is, does it matter whether students believe that their academic successes or failures are due to internal (ability or effort) or external (luck, professor, friends) factors?

To assess whether cultural differences in causal attribution habits contribute to self-efficacy, our study compares female college students from two countries: the USA and the Kingdom of Saudi Arabia (KSA). As a western country, the USA can be characterized as guided by individualistic culture; in contrast, the culture of KSA tends to emphasize collectivist values while also acknowledging the role of the individual (De Jong \& Moaddel, 2013; Haykel et al., 2015). KSA is a traditionally patriarchal conservative county ruled by the collectivist principles of Islam, but it is also a country that has strong Western influences and a younger generation that is somewhat bicultural because of increased modernization and second language education (e.g., English or French; Selvi \& Yazan, 2017). Female university students are selected for comparison because they are an understudied sample.

\section{Method}

The participants were 280 female students enrolled in a Core course (American Government) at the University of Central Florida in the USA, and 280 students enrolled in a Core course (Arabic Studies) at Prince Mohammed University in KSA. Participant ages ranged from 18 to 25. The KSA sample included students of Middle Eastern descent, whereas the USA 
sample included students from a more diverse ethnic background (White: 43.9\%; Hispanic: 26.8\%, Black: $11.8 \%$, Asian: 5.0\%, and mixed or unclassified: $12.5 \%$ ). As such, samples not only reflected cross-national differences in university enrollment, but also ensured the ecological validity of the study. Core courses, which are foundational courses taken by students irrespective of their major, were selected to ensure a sufficiently representative sample of students of each university. At the start of the course, students completed three questionnaires. The New General Self-Efficacy (NGSE; Chen et al., 2001) questionnaire assessed confidence in their abilities across a variety of competencies, behaviors, and situations. Students' agreement with each of the eight items of the questionnaire was measured on a 5-point Likert scale from 'strongly disagree' to 'strongly agree'. Cronbach's reliability coefficient (i.e., a measure of internal consistency) was .84.

The causal attribution questionnaire of McClure et al. (2011) assessed students' explanations of their best and worst performance (Cronbach's Alpha $=.60$ ). The questionnaire asked students to recall the time when they received either their best grade or worst grade on a test. Their task was to rate the contribution of seven causes to the respective grade on a scale from 0 (not at all) to 6 (entirely): ability, effort, test (either difficulty or ease), luck, family, instructor, and friends.

The cultural orientation questionnaire of Triandis and Gelfland (1998) measured students' orientation towards collectivism and individualism (Cronbach's Alpha $=.80$ ). The questionnaire assessed four types of beliefs about the self and others on a 9-point Likert scale from 0 (never) to 8 (always). It included vertical collectivism (VC; see oneself as a member of a hierarchically organized collective who is inclined to accept its inequalities), vertical individualism (VI; see oneself as independent who is inclined to accept its inequalities), horizontal collectivism (HC; see oneself as a member of a collective of equals among equals), and horizontal individualism (VC; see oneself as independent in a collective of equals among equals). On this scale, 4 was the neutral point.

General self-efficacy ratings were used to match each U.S. participant with a KSA participant on self-efficacy rating, thereby creating 280 pairs of U.S. -KSA participants. The subject pool from which selections were made included 1265 students. If a perfect match did not exist, the closest match was selected. When multiple candidates for a given match existed, random selection was used for pairing. The treatment of participants complied with the guidelines of the Office for Human Research Protections of the U.S. Department of Health and Human Services as well as the code of ethics of the American Psychological Association.

\section{Results}

All results described below were considered significant at the .05 level. When analysis of variance (ANOVA) was used, significant effects were followed by tests of simple effects, 
which were submitted to the Bonferroni inequality procedure to adjust for familywise alpha. Table 1 presents the descriptive statistics (mean, M, and standard error of the mean, SEM) of the main variables: self-efficacy, causal attribution preferences for desirable and undesirable outcomes, and cultural orientation.

Table 1. Descriptive statistics.

\begin{tabular}{lccccc}
\hline Variable & \multicolumn{2}{c}{ USA } & \multicolumn{2}{c}{ KSA } & \\
& Mean & SEM & Mean & SEM & Sign. \\
\hline Self-Efficacy & 3.03 & .031 & 3.03 & .034 & $n s$ \\
Best Grade & & & & & \\
Abilities & 4.80 & .067 & 4.03 & .069 & $<.001$ \\
Effort & 5.11 & .067 & 4.81 & .074 & .003 \\
Test & 3.06 & .084 & 3.32 & .098 & $n s$ \\
Luck & 1.88 & .096 & 2.27 & .110 & $n s$ \\
Family & 1.52 & .102 & 2.69 & .123 & $<.001$ \\
Instructor & 3.78 & .084 & 4.16 & .092 & .003 \\
Friends & 1.45 & .101 & 2.25 & .115 & $<.001$ \\
Worst Grade & & & & & \\
Abilities & 3.02 & .099 & 2.49 & .099 & $<.001$ \\
Effort & 4.21 & .097 & 2.97 & .110 & $<.001$ \\
Test & 4.37 & .075 & 4.34 & .097 & $n s$ \\
Luck & 1.55 & .094 & 2.14 & .114 & $<.001$ \\
Family & .73 & .074 & 2.00 & .126 & $<.001$ \\
Instructor & 2.93 & .099 & 3.65 & .112 & $<.001$ \\
Friends & 1.01 & .082 & 1.06 & .092 & $n s$ \\
Cultural Orientation & & & & & \\
HI & 6.244 & .0689 & 6.397 & .0677 & $n s$ \\
VI & 4.018 & .0830 & 4.439 & .0962 & .001 \\
HC & 5.788 & .0739 & 6.237 & .0824 & $<.001$ \\
VC & 5.430 & .0803 & 6.494 & .0858 & $<.001$ \\
\hline Vte. Signical
\end{tabular}

Note. Significant differences between U.S. and KSA students are displayed in the last column.

Participants' self-efficacy did not differ between countries, $F<1.00, n s$. Thus, the matching of participants on self-efficacy was judged successful. A 2 (country) X 7 (cause) mixed factorial ANOVA, conducted on the ratings for best grades, displayed a main effect of cause, $F(6,3348)=427.43, M S E=2.06, p<.001, \eta p^{2}=.434$, and a main effect of country, $F(1,558)$ $=16.02, M S E=4.66, p<.001, \eta p^{2}=.028$. There was also a significant interaction, $F(6,3348)$ $=28.56, M S E=2.06, p<.001, \eta p^{2}=.049$. Tests of simple effects (Bonferroni alpha: .007) indicated that, compared to KSA students, U.S. students preferred abilities and effort (internal causes) as explanations for desirable outcomes. In contrast, KSA students preferred family, friends, and the instructor (all external causes).

The same ANOVA performed on worst grades yielded a main effect of cause, $F(6,3348)=$ $350.19, M S E=2.43, p<.001, \eta p^{2}=.386$, and a main effect of country, $F(1,558)=3.05, M S E$ $=4.54, p=.005, \eta p^{2}=.005$. There was also a significant interaction, $F(6,3348)=40.40$, $M S E=2.43, p<.001, \eta p^{2}=.068$. Tests of simple effects (Bonferroni alpha: .007) indicated 
that U.S. students again preferred abilities and effort (internal causes) as explanations for undesirable outcomes. In contrast, luck, family, and the instructor (external causes) were preferred by KSA students. Thus, there were differences between countries, but U.S. students explained desirable and undesirable outcomes in the same way. KSA students also selected similar explanations for desirable and undesirable outcomes with one exception. To account for a desirable outcome, they saw friends as a potential agent of responsibility, but when an undesirable outcome was considered, they called upon luck.

Ranking the different explanations showed that U.S. students mentioned effort and ability as the most frequent accounts for desirable outcomes, and test difficulty and effort as the most frequent accounts for undesirable outcomes. Thus, U.S. students distinguished between different outcomes. For KSA students, desirable outcomes were preferentially attributed to effort and instructors, whereas undesirable outcomes were preferentially attributed to instructors and test difficulty. Thus, they were more likely to select an external cause than U.S. students to explain relevant outcomes.

A 2 (country) X 4 (cultural orientation) mixed factorial ANOVA conducted on ratings for cultural preferences displayed a main effect of cultural orientation, $F(3,1674)=401.58, M S E$ $=1.25, p<.001, \eta p^{2}=.418$, and a main effect of country, $F(1,558)=44.08, M S E=3.46, p$ $<.001, \eta p^{2}=.073$. There was also a significant interaction, $F(3,1674)=16.55, M S E=1.25$, $p<.001, \eta p^{2}=.029$. Tests of simple effects (Bonferroni alpha: .001) indicated that KSA students were more likely to endorse HC, VI and VC than U.S. students. Students from the U.S. and KSA displayed no differences in HI.

\subsection{Do explanations for best and worst grades account for self-efficacy?}

We carried out regression analyses for U.S. and KSA students separately to determine whether explanations for either best or worst grades, treated as the predictors, contributed differently to self-efficacy. Tables 2-3 illustrate the results of these analyses. Students from the U.S. and KSA differed in how they rated variables contributing to their self-efficacy. For U.S. students, self-efficacy benefited from the attribution of desirable outcomes to effort but was damaged by the attribution of desirable outcomes to luck. For KSA students, selfefficacy benefited from the attribution of desirable outcomes to abilities, whereas it was hurt by the attribution of a desirable outcome to the test difficulty. For U.S. students, self-efficacy was hurt by the attribution of undesirable outcomes to instructors. For KSA students, selfefficacy was hurt by the attribution of undesirable outcomes to abilities and luck. 
Table 2. Regression analyses for "best grade" with attribution preferences as the predictors, and self-efficacy as the outcome variable.

\begin{tabular}{lccccc}
\hline Best Grade-USA & $\boldsymbol{B}$ & $\boldsymbol{S E}$ & Beta & $\boldsymbol{t}$ & Sig. \\
\hline Constant & 2.283 & .192 & & & \\
Abilities & .049 & .027 & .107 & 1.790 & $n s$ \\
Effort & .088 & .027 & .194 & 3.198 & .002 \\
Test & -.011 & .023 & -.030 & -.489 &.$n s$ \\
Luck & -.044 & .021 & -.138 & -2.077 & .039 \\
Family & -.001 & .018 & -.003 & -.049 & $n s$ \\
Instructor & .039 & .022 & .107 & 1.745 & $n s$ \\
Friends & .022 & .020 & .073 & 1.113 & $n s$ \\
& & & & & \\
\hline Best Grade-KSA & & & & & \\
Constant & 2.302 & .144 & & & \\
Abilities & .155 & .027 & .352 & 5.722 & .000 \\
Effort & .033 & .026 & .080 & 1.273 & $n s$ \\
Test & -.047 & .020 & -.150 & -2.389 & .018 \\
Luck & -.022 & .017 & -.081 & -1.282 & $n s$ \\
Family & .026 & .015 & .104 & 1.747 & $n s$ \\
Instructor & .024 & .020 & .071 & 1.172 & $n s$ \\
Friends & -.011 & .017 & -.041 & -.652 & $n s$ \\
\hline
\end{tabular}

Note. USA: $R=.343$. KSA: $R=.437$.

Table 3. Regression analyses for "worst grade" with causal attribution preferences as the predictors, and self-efficacy as the outcome variable.

\begin{tabular}{lccccc}
\hline Worst Grade-USA & $\boldsymbol{B}$ & $\boldsymbol{S E}$ & Beta & $\boldsymbol{t}$ & Sig. \\
\hline Constant & 3.181 & .155 & & & \\
Abilities & -.031 & .018 & -.099 & -1.656 & $n s$ \\
Effort & .003 & .019 & .009 & .150 & $n s$ \\
Test & .024 & .026 & .059 & .926 & $n s$ \\
Luck & -.002 & .022 & -.007 & -.095 & $n s$ \\
Family & -.044 & .028 & -.106 & -1.562 & $n s$ \\
Instructor & -.039 & .020 & -.128 & -1.976 & .049 \\
Friends & -.029 & .026 & -.079 & -1.129 & $n s$ \\
\hline Worst Grade-KSA & & & & & \\
Constant & 3.212 & .111 & & & \\
Abilities & -.042 & .019 & -.137 & -2.224 & .027 \\
Effort & -.013 & .017 & -.048 & -.770 & $n s$ \\
Test & .022 & .021 & .070 & 1.065 & $n s$ \\
Luck & -.056 & .017 & -.209 & -3.273 & .001 \\
Family & .019 & .015 & .076 & 1.214 & $n s$ \\
Instructor & -.016 & .018 & -.058 & -.884 & $n s$ \\
Friends & .004 & .021 & .011 & .170 & $n s$ \\
\hline
\end{tabular}

Note. USA: $R=.246$. KSA: $R=.247$. 


\subsection{Does cultural orientation account for self-efficacy?}

We carried out regression analyses with self-efficacy as the outcome variable and cultural dimensions (HI, VI, HC, and VC) as the predictors, separately for KSA and U.S. students. Table 4 illustrates the results of these analyses.

Table 4. Regression analyses with cultural dimensions as the predictors, and self-efficacy as the dependent variable

\begin{tabular}{lccccc}
\hline Country & $\boldsymbol{B}$ & $\boldsymbol{S E}$ & Beta & $\boldsymbol{t}$ & Sig. \\
\hline USA & & & & & \\
Constant & 1.343 & .188 & & & \\
HI & .177 & .025 & .400 & 6.945 & .000 \\
VI & .018 & .019 & .049 & .924 & $n s$ \\
HC & .036 & .023 & .089 & 1.571 &.$n s$ \\
VC & .054 & .023 & .143 & 2.308 & .022 \\
KSA & & & & & \\
Constant & 1.877 & .187 & & & \\
HI & .127 & .028 & .281 & 4.462 & .000 \\
VI & .027 & .020 & .084 & 1.346 & $n s$ \\
HC & .058 & .027 & .158 & 2.165 & .031 \\
VC & -.023 & .027 & -.064 & -.841 &.$n s$ \\
\hline Note. & .372 & & & &
\end{tabular}

Note. USA: $R=.514$. KSA: $R=.372$.

For U.S. students, beliefs in horizontal individualism and vertical collectivism both contributed positively to self-efficacy. For KSA students, beliefs in horizontal individualism and collectivism contributed positively to self-efficacy. Thus, an appreciation for equity contributed to confidence in KSA students, whereas a mixture of conflicting orientations contributed to confidence in U.S. students.

\section{Discussion and Conclusion}

The present study examined the extent to which explanations for desirable and undesirable outcomes as well as cultural orientation accounted for self-efficacy. Our matched-pairs design suggested that both causal attribution habits and cultural orientation are related to several dimensions of self-efficacy even when students from different cultures are matched on their self-efficacy rating while holding sex constant (all participants were female). These findings are relevant to educators and administrators who wish to ensure that choices of curricula and instructional methods can adequately prepare students from different cultures for success. For example, U.S. students might be able to improve their self-efficacy if they are trained to attribute poor grades more to internal factors than to instructors. Further studies should look into how the context of a masculine culture such as that of KSA (see Hofstede 2001) may shape individuals' perception of self-efficacy. Comparing male students would also be of interest. 


\section{References}

Bandura, A. (1977). Self-efficacy: Toward a unifying theory of behavioral change. Psychological Review, 84(2), 191-215. https://doi.org/10.1037/0033-295X.84.2.191.

Bandura, A. (1989). Regulation of cognitive processes through perceived self-efficacy. Developmental Psychology, 25(5), 729-735.https://doi.org/10.1037/0012-1649.25.5.729.

Bartimote-Aufflick, K., Bridgeman, A., Walker, R., Sharma, M., \& Smith, L. (2016). The study, evaluation, and improvement of university student self-efficacy. Studies in Higher Education, 41(11), 1918-1942. https://doi.org/10.1080/03075079.2014.999319.

Chen, G., Gully, S. M., \& Eden, D. (2001). Validation of a new general self-efficacy scale. Organizational Research Methods, 4(1), 62-83. https://doi.org/10.1177/109442810141004.

Choi, I., Nisbett, R. E., \& Norenzayan, A. (1999). Causal attribution across cultures: Variation and universality. Psychological Bulletin, 125(1), 47-63. https://doi.org/10.1037/0033-2909.125.1.47.

de Jong, J., \& Moaddel, M. (2013). Trends in values among Saudi youth: Findings from values surveys. The Journal of the History of Childhood and Youth, 6(1), 151-164. https://doi.org/10.1353/hcy.2013.0015.

Haykel, B., Hegghammer, T., \& Lacroix, S. (Eds.). (2015). Saudi Arabia in Transition: Insights on Social, Political, Economic, and Religious Change. Cambridge University Press.

Hofstede, Geert H. (2001). Culture's Consequences. $2^{\text {nd }}$ ed. Sage.

Majer, J. M. (2009). Self-efficacy and academic success among ethnically diverse firstgeneration community college students. Journal of Diversity in Higher Education, 2(4), 243-250. https://doi.org/10.1037/a0017852.

McClure, J., Meyer, L. H., Garisch, J., Fischer, R., Weir, K. F., \& Walkey, F. H. (2011). Students' attributions for their best and worst marks: Do they relate to achievement? Contemporary Educational Psychology, 36(2), 71-81. https://doi.org/10.1016/j.cedpsych.2010.11.001.

Scholz, U., Doña, B. G., Sud, S., \& Schwarzer, R. (2002). Is general self-efficacy a universal construct? Psychometric findings from 25 countries. European Journal of Psychological Assessment, 18(3), 242-251. https://doi.org/10.1027//1015-5759.18.3.242.

Selvi, A. F., \& Yazan, B. (2017). English as an international language pedagogy: A sustainable alternative for teaching English in the GCC region. In A. Mahboob \& T. Elyas (Eds.), Challenges to education in the GCC during the 21st century, (pp. 65-90). Gulf Research Center.

Triandis, H. C. \& Gelfand, M. J., \& (1998). Converging measurement of horizontal and vertical individualism and collectivism. Journal of Personality and Social Psychology, 74(1), 118-128. https://doi.org/10.1037/0022-3514.74.1.118.

van Dinther, M., Dochy, F., \& Segers, M. (2011). Factors affecting students' self-efficacy in higher education. Educational Research Review, 6(2), 95-108. https://doi.org/10.1016/j.edurev.2010.10.003. 